\title{
Biopsychosocial model for a multidisciplinary perioperative care pathway in patients undergoing posterior spinal fusion surgery for adolescent idiopathic scoliosis
}

Spaas C, de Backer S, Gios J, Hans G, Saldien V, Breebaart M, Sermeus L, Michielsen J, Wildemeersch D Antwerp University Hospital Belgium, Department of Anesthesia \& Pain Center and Orthopedic Surgery

\section{Background}

Adolescent idiopathic scoliosis (AIS) is the most common form of scoliosis, mostly affecting children. Although AIS is a benign disorder with no severe long-term consequences, literature suggests that besides worse pain, self-image is lower among cohorts with untreated AIS.

Posterior spinal fusion (PSF) for AIS is an invasive surgical procedure associated with severe postoperative pain, it has a challenging pain management and patients are at risk for persistent postoperative pain.

\section{Aim}

The purpose of this study is the implementation of an enhanced recovery pathway (ERP) for AIS patients undergoing PSF including all aspects of integrated biopsychosocial care (Figure.1).

\section{Methods}

- Systematic literature search

\section{- Critical appraisal of the current perioperative standardized} multimodal analgesic protoco

\section{- Considering all aspects of perioperative patient care}

- Evaluation of several scores for their potential to quantify biopsychosocial elements that reflect patient wellbeing

Primary outcome parameter. postoperative pain (NRS, numeric rating scale)

Secondary outcomes: opioid-related side effects (nausea, vomiting and pruritus and mobilization onset)

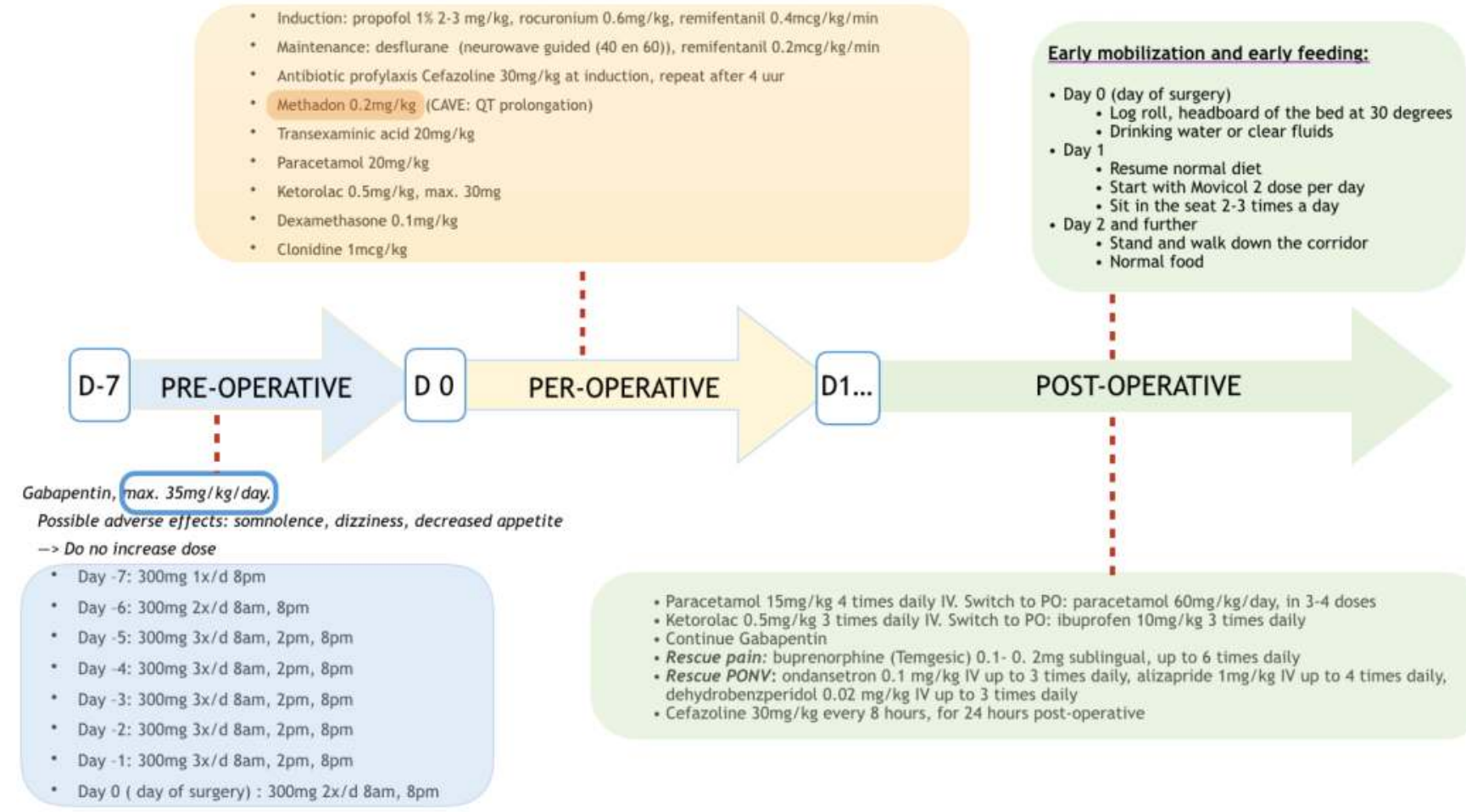

Fig 2. Flowchart peri-operative management

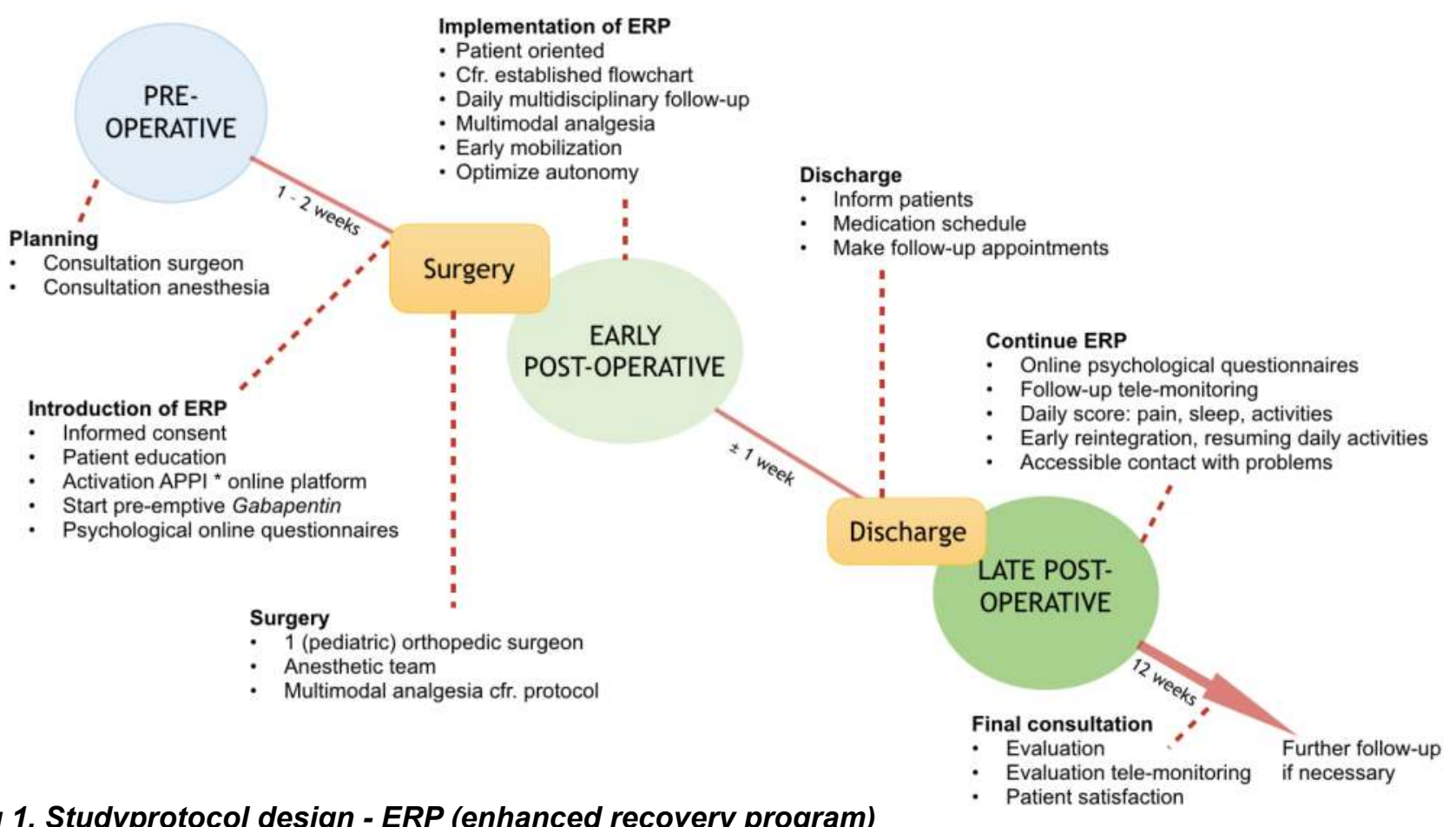

\section{Results and discussion}

"Bio" treatment model

- New multimodal analgesic protocol (Figure 2.):

- preemptive oral gabapentin (to start 7 days prior to surgery and continue postoperatively)

Anti-epileptic drug, important role in the treatment of neuropathic pain syndromes, important opioid-sparing effect.

• no (patient controlled) epidural analgesia

- single intraoperative dose of methadone IV

Potent $\mu$-receptor agonist, inhibitory effect on the $N$ - methyl-D-aspartic acid (NMDA) receptors involved in the development of opioid tolerance, hyperalgesia and the development of chronic pain. In addition, it inhibits the reuptake of serotonin and norepinephrine and thus plays a role in the in antinociception and mood elevation.

- avoid use of morfine or tramadol perioperatively

- standard use of acetaminophen and NSAID perioperatively

- buprenorphine sublingually on demand postoperatively

- anti-emetic prophylaxis peroperatively (dexamethasone) and treatment posteroperatively (ondansetron, alizapride, dehydrobenzperidol)

- clonidine peroperatively

Additive opioid-sparing and analgesic effect

Psychosocial care path

- Psychological preoperative screening using Web-based questionnaires

Screening for and if necessary treatment of yellow flags (catastrophic behavior, anxiety, kinesiophobia, etc.).

- Extensive information and education about surgery and postoperative expectations

Anxious patients are at risk of more severe acute postoperative pain, chronification of pain and poor coping mechanisms.

\section{- Extensive patient related outcome measure registration}

- Long-term follow up using eHealth based telemonitoring devices and online daily questionnaire

\section{Conclusion}

- Study design with implementation of a biopsychosocial model in a multidisciplinary perioperative care pathway for adolescents undergoing posterior spinal fusion

- Implementation of ERP could result in reduction of postoperative pain and opioid-related side effects, earlier hospital discharge and may improve patient satisfaction 